\title{
Philosophiques
}

\section{C.-G. Jung et le féminin}

\section{Raynald Valois}

Volume 21, numéro 2, automne 1994

Les femmes et la société nouvelle

URI : https://id.erudit.org/iderudit/027281ar

DOI : https://doi.org/10.7202/027281ar

Aller au sommaire du numéro

Éditeur(s)

Société de philosophie du Québec

ISSN

0316-2923 (imprimé)

1492-1391 (numérique)

Découvrir la revue

Citer ce document

Valois, R. (1994). C.-G. Jung et le féminin. Philosophiques, 21(2), 393-404.

https://doi.org/10.7202/027281ar
Résumé de l'article

L'anthropologie jungienne propose un modèle de réalisation psychologique dont on n'a pas encore mesuré la portée en regard des futurs rapports homme-femme. On peut même soutenir que la psychologie jungienne concerne davantage l'humanité qui se profile à l'orée du XXIe siècle, que les sociétés actuelles les plus avancées, et cela spécialement en regard de l'intégration de la composante féminine dans la psyché humaine totale. d'utilisation que vous pouvez consulter en ligne.

https://apropos.erudit.org/fr/usagers/politique-dutilisation/ 


\title{
C.-G. Jung et le féminin
}

\author{
par \\ Raynald Valois
}

\begin{abstract}
RÉSUMÉ : L'anthropologie jungienne propose un modèle de réalisation psychologique dont on n'a pas encore mesuréla portée en regard des futurs rapports homme-femme. On peut même soutenir que la psychologie jungienne concerne davantage l'humanité qui se profile à l'orée du XXI siècle, que les sociétés actuelles les plus avancées, et cela spécialement en regard de l'intégration de la composante féminine dans la psyché humaine totale.
\end{abstract}

ABSTRACT : Jung's anthropology offers us a model of psychological fulfillment the importance of which has not yet been realized with respect to the future relations between men and women. It is even possible to argue that Jung's psychology will be of more concern to the next century, because of its integration of the feminine part of the human psyche.

Mon intention n'est pas d'exposer, même très sommairement, les positions de Jung sur la psychologie féminine. Je veux simplement attirer votre attention sur certains points qui me semblent particulièrement intéressants en regard des interrogations actuelles de la pensée féministe. En effet la nature du psychisme féminin et ses interrelations avec le masculin occupent une position si fondamentale chez ce penseur, que l'on peut difficilement en traiter comme d'un chapitre particulier de son œuvre. Je crois cependant que certains aspects de sa psychologie sont de nature à projeter un éclairage original sur les questions qui nous préoccupent actuellement.

C'est évidemment d'un point de vue philosophique que je m'intéresse à Jung. En ceci je ne crois cependant pas récupérer l'œuvre de ce grand psychologue pour des fins étrangères à ses propres préoccupations. Il reprochait luimême à la philosophie actuelle de ne pas encore avoir reconnu l'inconscient comme un facteur autonome opposé au conscient, aussi important que lui et capable d'interférer avec la conscience au moment qui lui plaît ${ }^{\mathrm{I}}$. De même, lorsqu'il découvrit la psychiatrie, à la fin de ses études médicales, il se fit la

I. C.G.Jung parle, rencontres et interviews, Editions Buchet/Chastel, Paris, 1985, p. 266. 
réflexion suivante : «C'est le confluent de la médecine et de la philosophie ! C'est ce quej'ai toujours cherché2 ${ }^{2}$ »

Le fait est que Jung est plus qu'un psychologue au sens ordinaire du mot. Depuis Kant, et même peut-être depuis Descartes, la philosophie a comme objet principal la critique de la connaissance : « Que vaut notre image du monde ? », telle est la grande question. En réfléchissant sur les arrière-plans inconscients qui alimentent et fondent cette image, Jung se livre à une tâche hautement philosophique. Entre autres, ses théories concernant l'état de participation mystique originelle, qui recule graduellement avec le développement de la conscience, nous obligent à la plus grande prudence en ce qui concerne les limites de la connaissance rationnelle. Cet état d'identité à l'objet qui fait que ce dernier est totalement ou partiellement masqué par les images que nous y projetons, n'est en effet reconnu qu'une fois que la conscience a surmonté l'inconscience. La fierté que nous retirons devant les idoles que notre savoir a abattues ne peut donc éclipser nos incertitudes au sujet de celles qui continuent sans doute à nous aveugler. Comme exemple de ceci, nous venons tout juste de voir s'écrouler le mythe du paradis socialiste : quelle sera la prochaine victime de l'évolution de la pensée?

Jung est donc un épistémologue, un philosophe de la connaissance, et pas seulement un médecin des émotions. Bien plus, si, comme il le soutient, notre vision du monde est issue des formes a priori contenues dans l'inconscient collectif, explorer ce dernier, n'est-ce pas rechercher les fondements ultimes du réel, ce qui est une tâche proprement métaphysique? Comme le dit la sagesse indienne : "Qui se connaît lui-même connaît et le Monde et Dieu », ce qui n'est pas si loin du « Connais-toi toi-même » de Socrate.

D'après ce que nous livre l'histoire des religions, il fut un temps où le Monde apparaissait comme engendré par la Grande Déesse Mère, et dominé par les forces fêminines. Le principe premier était alors la matière, la « matermateria », tandis que l'esprit ne représentait que son fruit : la nuit engendrait le soleil et non l'inverse. Puis vinrent les conceptions patriarcales : Athéna sortait maintenant de la tête de Zeus, le cosmos de la bouche de Yaveh, et Ève de la côte d'Adam.

Voilà quelques exemples de la façon dont les structures profondes de la psyché humaine imposent à une conscience historique donnée une certaine façon de se représenter et de s'expliquer l'univers auquel elle est confrontée. La raison s'empare ensuite de ces images, les analyse, les structure, les organise en système et construit des métaphysiques. Ainsi se trouve-t-il que la philosophie n'est peut-être pas aussi asexuée qu'on pourrait le croire. Peut-être même qu'une réévaluation de l'importance relative des principes masculins et féminins pourrait résulter en une nouvelle vision du monde pleine de

2. Ibid., P. 166. 
surprises pour notre tradition patriarcale. Voilà certaines perspectives que nous ouvrent les réflexions de Jung sur les structures et le fonctionnement de la psyché.

\section{La psyché est bisexuée}

Un premier point que je veux souligner, c'est le caractère bisexué de la psyché humaine. Dans la lutte pour l'égalité des sexes, on a souvent tendance à réduire ces derniers aux différences anatomiques et physiologiques. Seuls les corps seraient différents et complémentaires en vue de la reproduction. Les autres caractéristiques ne seraient que le résultat de stéréotypes sociaux imposés par la division des tâches et la domination masculine traditionnelles.

Au contraire, pour Jung, l'opposition entre le féminin et le masculin constitue la base même du psychisme humain. L'examen des oppositions qui structurent la psyché nous révèle en effet qu'elles ont toutes une grande affinité avec celle des sexes, chacun des contraires ayant toujours un caractère soit masculin soit féminin.

Cette façon de voir repose en premier lieu sur des observations cliniques comme l'analyse des rêves et des rêveries éveillées des nombreux patients et patientes que Jung a traités durant sa très longue carrière (Igoo à Ig6I) : il affirmait avoir analysé plus de 80,000 rêves. De plus, l'étude des mythologies venait confirmer et élargir ses théories. Une des hypothèses fondamentales de Jung consiste en effet à poser que les mécanismes psychiques qui sont à l'origine de la création des mythes et des rites universels sont les mêmes chez tous les hommes et continuent à alimenter l'imaginaire moderne. La connaissance de ces mythes nous permet donc de psychanalyser nos lointains ancêtres, comme les rêves et les fantasmes de nos contemporains guident le psychologue actuel dans son travail clinique. Or il est évident qu'une des grandes structures de la mythologie consiste dans l'opposition et la lutte entre divinités masculines et féminines. Il semble donc que telle aussi soit la structure de l'inconscient collectif qui habite tous les humains.

En fait les différents complexes qui constituent les noyaux énergétiques qui polarisent l'action de l'inconscient sont toujours personnifiés, aussi bien dans les rêves que dans les mythes, par des hommes et des femmes, ou par des animaux associés respectivement aux divinités masculines et féminines.

Ceci n'est pas surprenant puisqu'une autre hypothèse de base de Jung veut que toute l'organisation énergétique de la psyché repose sur l'opposition entre des pôles contraires entre lesquels circule cette énergie qu'il a d'abord nommée libido, selon le vocabulaire freudien, puis tout simplement énergie psychique, à cause de la signification trop unilatéralement sexuelle du premier terme ${ }^{3}$. Sans tension entre des forces contraires, pas de transfert d'énergie, 
donc pas d'action : inertie totale. Lorsque les différences de potentiel sont nivelées, c'est la suspension ou l'arrêt du mouvement vital.

Lorsque la conscience est trop unilatéralement dominée par l'un des pôles, il se produit donc nécessairement dans l'inconscient une activation compensatoire du pôle contraire en vue de retrouver une situation d'équilibre, c'est-à-dire une situation ou l'énergie circule librement entre ces pôles. Ainsi un homme exclusivement orienté selon ses tendances masculines et refoulant toute attitude féminine risque de souffrir de troubles émotionnels et de symptômes dont le but ultime est de rétablir l'équilibre de sa personnalité ${ }^{4}$.

Ceci signifie que les composantes psychiques masculines et féminines sont simultanément présentes aussi bien dans la femme que dans l'homme, ce qui est une certaine forme d'androgynie. Cependant il y a une différence importante : chez la femme, la masculinité est au départ inconsciente et chez l'homme il en va de même pour la féminité. C'est le phénomène auquel Jung a donné les noms bien connus d'anima et d'animus. La féminité inconsciente de l'homme s'exprime dans la figure de l'anima, qu'il projette facilement sur les femmes qui l'entourent, tandis que la femme fait de même avec son animus. En termes freudiens, on dira que l'homme prend la femme pour sa mère et que sa partenaire le prend pour son père, ce qui n'est pas très différent de la position jungienne, car aux traits féminins impersonnels et universels de l'anima et de l'animus viennent nécessairement s'adjoindre les déterminations résultant de la relation au père et à la mère personnels.

Pour bien comprendre cette problématique et ne pas en rester au niveau des généralités, il faut cependant aller plus loin et nous poser la grande et terrible question : quels sont ces traits psychologiques que l'on peut considérer comme naturellement propres au masculin et au féminin ? Jung a beaucoup disserté sur le sujet et l'un de ses disciples, Erich Neumann, s'est livié à une étude très documentée des symboles masculins et féminins à travers les grandes mythologies aux quatre coins du monde. Je vais donc tenter de vous donner un aperçu de leurs observations, en mettant, comme Neumann, l'accent sur le féminin.

\section{L'ouroboros}

Dans le Timée $e^{5}$ Platon raconte que le monde originel était vivant, composé de quatre éléments, sphérique, doté du mouvement circulaire, divin, possédant une âme. Plus généralement le grand rond, qu'il soit figuré comme ouroboros (le serpent ou le dragon qui se mord la queue), comme lotus, comme œuf cosmique ou toute autre forme circulaire ou sphérique est un symbole universel de l'état originel du cosmos. Or il se trouve que tous les mythes

4. Voir, entre autres, la section L'anima et l'animus dans Dialectique du Moi et de l'nnconscient, Gallimard, Paris, 1964, p. 137-191.

5. $2 g \mathrm{~b}-34 \mathrm{~d}$. 
originels ne sont qu'une traduction imagée à travers laquelle nos ancêtres les plus lointains percevaient la naissance de leur propre conscience. Or, comme sur le plan biologique, chaque individu récapitule, dans sa propre genèse psychique, celle de l'humanité. Jung a donc émis l'hypothèse que le stade initial de la psyché en serait un d'auto-suffisance, de complétude, d'indifférenciation, c'est-à-dire de mélange dans lequel tous les contraires sont présents et agissants de façon chaotique : dans l'ouroboros les extrêmes se touchent, se dévorent et se fécondent mutuellement. Il est intéressant de noter, en passant, que la cosmologie la plus récente propose comme stade originel du cosmos un état analogue d'indifférenciation, une grande soupe, comme dit Hubert Reeves, (les Indiens parlaient de brassage de lait : les recettes ont changé mais l'idée est la même) dans laquelle on ne pourrait pas encore distinguer même des particules très élémentaires.

Évidemment ce stade en est aussi un d'androgynie, ce qui est aussi très souvent symbolisé par des divinités originelles, androgynes. Par exemple, dans le Banquet ${ }^{6}$, Platon décrit un androgyne originel, rond, se déplaçant d'un mouvement circulaire et doté d'une force qui lui permettait de dēfier les dieux. Ceci signifie donc que la psyché, aussi bien celle de l'homme que celle de la femme, est à l'origine dans un état complet d'indifférenciation sexuelle.

Un autre aspect du mythe consiste en ce que la lumière n'a pas encore été séparée des ténèbres, ce qui signifie que la conscience n'a pas encore été séparée de l'inconscient: il n'y a encore aucune possibilité de connaissance de soi, parce qu'il n'y a pas encore cette séparation entre un Moi, un Ego, et un Soi vers lequel cet Ego puisse se retourner comme vers un Autre, ce en quoi consiste la réflexion.

\section{La Grande Mère}

La phase ultérieure du développement est représentée par l'émergence de la lumière, ce qui constitue la création à proprement parler et le prélude à l'apparition de l'être humain, lequel symbolise l'émergence du Moi conscient. Avec la séparation de la lumière et des ténèbres, du ciel et de la terre, on assiste à une première différenciation des sexes sous formes des Parents originels. C'est aussi à ce moment que les traits psychologiques propres à chacun commencent à prendre de la consistance.

La femme dans son aspect de Déesse Mère se voit alors identifiée à la terre, source et origine de toute vie : tous les êtres sortent de son sein, sont nourris par elle puis finalement repris en son sein dans le sommeil de la mort. Autour de ce symbole central gravite une foule d'images qui expriment les différents aspects de la féminité. À titre d'exemple Jung en dresse une liste sommaire qui est déjà fort impressionnante: 
[...] la terre, la forêt, la mer et l'eau tranquille; la matière, les enfers et la lune; [...] le champ, le jardin, le rocher, la grotte, l'arbre, la source, le puits profond, les fonds baptismaux, la fleur considérée comme vase (rose et lotus) [...] le four, la marmite, sous la forme animale, la vache, le lièvre et, en général tout animal secourable. [...] l'Eglise, l'université, la ville, le pays... ${ }^{7}$

Il explique ensuite que tout ce symbolisme est ambivalent: il y a d'un côté la Bonne Mère, celle qui engendre, nourrit, protège, entoure de soins, soutient, fait croître, tous traits qui nous sont familiers; mais d'un autre côté, il y a la Mère Terrible, celle qui dévore ses enfants, le monstre qui avale le héros, la déesse de la guerre et de la mort, celle qui castre et tue ses fils-amants.

En termes psychologiques la Mère représente l'inconscient en tant que source et matrice de toute vie consciente. De plus, le symbolisme végétal et animal dont elle s'entoure montre aussi l'affinité de cette figure avec la sphère des instincts. Ce portrait s'accorde aussi avec la conception chinoise pour qui le yin correspond au côté obscur, froid et humide de la montagne tandis que le yang masculin est clair, chaud et sec. Bien plus, l'état cahotique et indifférencié de l'ouroboros, dont les principaux symboles se moulent dans des formes circulaires et englobantes, témoigne évidemment d'une prédominance du féminin, malgré son essence androgyne. Comme le dit Lao Zi du Tao :

Un être s'est formé.

Il est né avant le ciel et la terre;

Seul, indépendant, immuable;

Partout en mouvement, immortel.

Peut-être est-il la mère du monde.

Je ne sais pas son nom,

Je l'appelle Tao ${ }^{8}$.

Or on sait que pour Jung, le Tao n'est rien d'autre que l'inconscient collectif, source et fondement de toute vie psychique. Comme je l'ai dit, l'inconscient est à la fois masculin et féminin. Cependant en tant que force génératrice et conservatrice de la vie psychique, il a un caractère nettement féminin-maternel.

Dans son aspect positif cette force est absolument essentielle et fondamentale. C'est elle qui assure à l'être humain son enracinement dans les lois de la nature, celles qui assurent la santé et l'équilibre mental.

A première vue il ne semble pas très glorieux pour la femme d'être associée à l'obscurité de la nuit. Évidemment, dans la lumière, la chaleur, la clarté masculine de la conscience, c'est beaucoup plus brillant, c'est le cas de le dire. Pourtant les mythes racontent que c'est la nuit qui a donné naissance au jour, et, même avant que le soleil ne se lève, elle est déjà peuplée d'une nuée d'étoiles, d'une infinité de petits soleils. Il y a une sagesse de la nuit, de la terre,

7. Les Racines de la conscience, Ed. Buchet/Chastel, Paris, 1971, p. 96-97.

8. Le Tao et la vertu, Trad. Joseph Liu, Ed. Parti pris, Montréal, 1974, p. 78. 
de la nature, beaucoup plus profonde que celle de la Raison. C'est le monde de l'imaginaire, de l'inspiration créatrice, la mère de toutes les grandes réalisations. C'est pourquoi on peut affirmer que toutes les grandes conquêtes de la culture : la religion, la philosophie, et l'art, ne sont que des fruits portés par l'arbre féminin de l'inconscient.

Notre civilisation s'est édifiée sur une valorisation unilatérale des aspects masculins de la psyché. Le mythe d'Icare symbolise bien notre tragique destin : au moyen d'ailes soudées avec de la cire nous avons voulu nous détacher de la Terre et voler toujours plus près du Soleil. Nous sommes hypnotisés par l'attrait de la lumière intellectuelle : science, technique, acier, verre et béton. Mais les ailes d'Icare ont fondu et il a fait une chute vertigineuse dans la Mer. Heureusement, celle-ci est maternelle et peut-être qu'après l'avoir englouti elle le rendra à une vie nouvelle comme Jonas dans la baleine.

En quoi consiste cette sagesse féminine, fille de la nuit? Elle a toujours été identifiée à un pouvoir magique qui donne accès à l'au-delà, aux inspirations surnaturelles, à la divination sous toutes ses formes. Même Socrate, que Nietzsche accuse d'avoir tué la poésie, a fait l'éloge du délire, en se référant à la prophétesse de Delphes, aux prêtresses de Dodone, à la Sibylle:

Autant donc l'art divinatoire l'emporte évidemment en perfection [...] autant le délire, au témoignage de l'Antiquité, est une chose plus belle que le bon sens : le délire qui vient d'un Dieu, qu'un bon sens dont l'origine est humaine ${ }^{9}$.

Il est intéressant de noter que Socrate associe au délire l'art de deviner l'avenir, l'art de la guérison, celui de la poésie, inspirée par les Muses, et enfin l'amour de la Beauté qui fait pousser des ailes à l'âme et l'élève vers la contemplation des réalités absolues ${ }^{\text {IO }}$. Et voilà que l'Éros féminin entraîne l'Âme vers les sommets de la Sagesse. Si la Sagesse est femme, l'amour de la Sagesse, la philo-Sophie, serait peut-être aussi affaire de femme.

\section{Éros et logos}

Jung voit justement dans l'Éros le trait le plus caractéristique de la féminité :

La psychologie de la femme repose sur le principe du grand Éros qui unit et sépare, tandis que l'homme s'attache, depuis toujours, au Logos comme principe suprême. En langage moderne, on pourrait traduire le concept de l'Eros par : « relation spirituelle » et celui du Logos par « intérêt objectif »"

Et un peu plus loin Jung précise ce qu'il entend ici par « spirituel » en l'associant à « inconscient ». Or l'inconscient, c'est cette totalité originelle à partir de laquelle est engendré le Moi comme un Fils divin. L'Éros représente donc cette force de cohésion qui travaille à maintenir l'unité et l'intégrité de la

\footnotetext{
9. Phedre, $244 \mathrm{~d}$.

Io. Ibid., 249d sq.

II. Problèmes de l'âme modene, Ed. Buchet/Chastel, Paris, I976, p. 286.
} 
psyché. C'est une force centripète qui attire toutes les parties vers le milieu et préserve de l'éclatement. C'est encore l'Éros qui tend à maintenir la pensée rationnelle dans la continuité de ses racines inconscientes grâce aux liens entretenus par l'intuition. Combien d'œuvres philosophiques auraient gagné en substance à s'alimenter dans l'intuition? Que de verbiages creux nous seraient épargnés? Le fait est que l'inconscient a la vue moins claire que le conscient mais combien plus large et plus profonde. Si l'étroitesse et l'unilatéralité de la pensée consciente ne sont pas compensées par un accès très libre aux trésors de l'inconscient, celle-ci perd rapidement contact avec le réel et s'égare dans des constructions, logiques en apparence, mais sans véritable intérêt pour l'acquisition de la sagesse, qui doit être englobante.

Ce qui est vrai de la philosophie et de la pensée en général, l'est encore plus en ce qui concerne la conduite de la vie personnelle et l'adaptation au monde qui nous entoure. J'ai mentionné plus haut que le symbolisme féminin renvoyait très souvent à la vie animale et instinctuelle : dans beaucoup de mythologie la femme est figurée comme la Reine des animaux. Cette dimension de la psyché se trouve hautement méprisée dans notre civilisation qui a homologué le corps, la sexualité et aussi la femme (la sorcière) au mal et à Satan. Il en résulte un déracinement très dangereux, une sorte de dissociation de l'âme moderne, qui n'a cessé de préoccuper Jung tout au long de sa carrière. Le patriarcat occidental a hypertrophié à un point tel l'aspect rationnel-masculin de la psyché, qu'il nous a coupé de la sphère des instincts qui sont nécessaires à notre survie individuelle et collective. C'est ainsi que l'on en est arrivé à se construire un mode de vie complètement artificiel, qui conduit des foules de gens à ruiner aussi bien leur santé physique que leur équilibre émotionnel. La dévastation de l'environnement n'est qu'un symptôme parmi d'autres de l'insensibilité moderne à tout ce qui est nature et vie.

\section{La mère terrible}

L'aspect négatif de l'image de la Mère originelle, c'est principalement son caractère dévorant. D'après Erich Neumann, les ogres et les ogresses, les monstres de toutes sortes qui menacent les héros des mythes et des légendes ont toujours un lien étroit avec la Grande Déesse Mère.

Psychologiquement parlant, il est alors question de la force d'attraction que l'inconscient exerce sur le conscient. Comme je l'ai déjà mentionné, toute la vie psychique se joue comme une lutte entre des forces contraires. L'instinct de conservation, qui veut s'assurer que l'individu ne coupera pas le contact avec ses racines et ne se perdra pas dans des attitudes et des comportements inadaptés ou de nature à mettre sa vie en danger, peut entraver son développement et l'emprisonner dans une sorte d'inertie psychique.

Dans les mythes, la victoire du Moi sur ces forces menaçantes est symbolisée par la victoire du Héros masculin sur le monstre féminin. Dans cette lutte épique, le Héros est supporté par le Père divin spirituel. Ceci signifie, pour Erich Neumann, que les forces psychiques qui soutiennent la 
formation du Moi individuel, de la conscience personnalisée, ont un caractère masculin, aussi bien chez la femme que chez l'homme. C'est d'ailleurs aussi ce que pense le freudisme en soutenant que c'est le père qui coupe le cordon ombilical qui retient et le fils et la fille à la mère et à l'état d'inconscience et d'irresponsabilité infantile.

Lorsque le Héros échoue dans son entreprise, comme c'est le cas d'Attis, d'Adonis, de Dionysos, d'Osiris, etc., il meurt dans la fleur de l'âge, castré, démembré, et dévoré par la Grande Mère ou ses suppôts. Cela signifie que l'attraction de l'inconscient a réussi à empêcher le Moi de se former en tout ou en partie. La mort du Moi c'est, ou bien la stagnation dans un état d'inconscience et d'immaturité, ou, dans les cas graves, la psychose. La mort du Héros peut cependant signifier le passage par un état ténébreux et souffrant qui sera suivi d'une résurrection, d'un nouvel enfantement. Alors le sein de la Grande Mère devient un vase de transformation.

\section{L'arbre philosophique}

Quand le Moi a réussi à émerger, aussi bien chez l'homme que chez la femme, ce qui correspond à l'acquisition du statut d'adulte, l'évolution psychologique est loin d'être terminée. En effet, la victoire de la lumière sur les ténèbres, du conscient sur l'inconscient, la séparation de la terre maternelle, la sortie du Soleil de l'Océan cosmique et son élévation au zénith, cet acquis n'est jamais définitif. Le Soleil devra replonger à nouveau dans ces mêmes ténèbres, être de nouveau avalé par le monstre marin et renaître au matin encore plus vigoureux qu'auparavant, et cela selon un cycle perpétuel comme celui des jours et des saisons.

Nous abordons ici le thème qui contrarie sans doute le plus carrément notre moderne mentalité. Notre civilisation prométhéenne se glorifie d'avoir ravi le feu aux divinités de l'Olympe, c'est-à-dire aux forces de l'inconscient. Fière d'avoir instauré le royaume de la déesse Raison, elle n'a que faire des intuitions, des pressentiments, des émotions et autres faiblesses féminines, comme si la domination de la lumière, de la chaleur et du sec pouvait être définitive. L'avance implacable du désert et le recul constant de la forêt vierge symbolisent bien au plan planétaire cette maladie psychique de notre civilisation.

Pourtant le but ultime du développement humain est symbolisé dans toutes les traditions par un retour à la totalité et à l'unité de l'androgyne originel, réalisées désormais à un niveau supérieur de la conscience. Ce processus psychologique d'aspect tout à fait paradoxal, implique une communion très étroite entre les forces féminines de l'inconscient, toutes en largeur et en profondeur et l'intensité masculine, fondée sur l'étroitesse et la concentration. En effet la conscience claire est toujours limitée comme un faisceau très mince qui permet d'être présent à l'ici et maintenant, tandis que la lumière aurorale de l'inconscient se déploie à l'infini dans le passé et l'avenir, l'ici et l'ailleurs, et 
rend possible une orientation appuyée sur la perception globale de nos horizons psychiques.

Ce processus d'intégration des deux moitiés de la psyché a été appelé individuation, par Jung. Il lui a consacré la majeure partie de ses recherches, parce qu'il y a vu le problème le plus grave du $\mathrm{XX}^{\mathrm{e}}$ siècle, celui qui risque de conduire et l'individu et l'humanité au seuil de l'abîme. La quête des sources de l'imagerie inconsciente de l'âme moderne l'a conduit à fouiller les mouvements de pensée qui ont mené une vie souterraine depuis le début de la chrétienté et se sont maintenus malgré tous les anathèmes de l'autorité cléricale, à savoir la gnose et la philosophie alchimique. En effet, il y a découvert une prolifération de symboles, où il a discerné une immense réaction compensatoire à l'unilatéralité du patriarcat chrétien. Il a pu, grâce à de multiples études comparatives, déterminer la signification de ce symbolisme et arriver à la conclusion que le Grand Euvre alchimique n'avait rien à voir avec la transmutation physique de la matière et la fabrication de l'or. Le but visé inconsciemment consistait à intégrer dans le développement psychique l'aspect matériel et ténébreux, refoulé par un christianisme uniquement préoccupé de spiritualiser l'homme. L'opération en était une de croissance intérieure et de conciliation des contraires : d'où l'idée de noces alchimiques et d'enfantement de l'androgyne.

Or les trois plus grands symboles de l'ceuvre sont le mercure, l'athanor (vase dans lequel on opérait) et l'arbre philosophique. Le mercure est une substance extraite du sein de la terre mère et qui représente son esprit double, masculin et surtout féminin; le vase n'est autre que l'utérus dans lequel s'opère la transmutation; enfin l'arbre représente le processus de croissance qui doit amener l'individu à sa pleine maturité. C'est aussi un symbole féminin parce qu'il renvoie au dynamisme intérieur, aux forces de la fécondité naturelle qui poussent la psyché dans la direction de son achèvement. Voici comment Jung résume le symbolisme de l'arbre:

Les associations statistiquement les plus fréquentes concernant le sens de l'arbre sont probablement la croissance, la vie, le déploiement de la forme au point de vue physique et spirituel, le développement, la croissance de bas en haut et vice versa, l'aspect maternel : protection, ombrage, toit, fruits comestibles, source de vie, fermeté, durée, enracinement let aussi impossibilité de bouger de place), âge, personnalité et enfin mort et renaissance ${ }^{12}$.

Une magnifique illustration de ce symbolisme nous est fournie par une image empruntée à la mythologie germanique. On y voit Yggdrasill, l'arbre cosmique de l'Edda, qui plonge ses racines dans les profondeurs du monde souterrain, des enfers, traverse le disque de la terre en son centre pour se déployer dans la voûte céleste, comme une puissance protectrice qui dispense la vie à tous les êtres.

12. Les racines de la conscience, p. 352. 
En bref, tout ce symbolisme met en grande évidence le fait que l'évolution psychologique à laquelle est désormais confronté l'adulte n'est pas principalement une affaire masculine de volonté, de cogitations, de décision, comme chez l'adolescent, mais plutôt une capacité de prêter l'oreille à la Voix intérieure, à cette poussée venue du dedans qui se traduit par toutes sortes de messages lancés par l'inconscient : rêves, fantasmes, obsessions, pour ne rien dire de tous les troubles physiques qui nous obligent à nous arrêter vers le milieu de la vie.

On peut conclure de tout ceci que la féminité n'est pas simplement un principe de conservation et d'inertie, mais aussi un facteur dynamique qui conduit la psyché à son accomplissement ultime, à l'intégration finale qui doit rétablir l'unité qui a dû être temporairement sacrifiée pour permettre au Soi de devenir conscient de lui-même. D'où il devient clair que, lorsqu'on parle de passivité au sujet du principe féminin, il faut bien comprendre qu'il s'agit de cette disponibilité de la terre à être labourée et fécondée par le principe masculin. Mais il faut surtout se rendre à l'évidence que c'est elle qui fait pousser la plante et que c'est elle, en tant que nature, qui agit à l'intérieur de celle-ci pour la mener à maturité. À la limite la terre pourrait s'arranger seule, sans l'intervention souvent maladroite et désastreuse de celui qui prétend améliorer le rendement.

\section{Conclusion}

Je suis bien conscient que ces quelques réflexions ne peuvent donner qu'une idée bien sommaire des trésors qui ont été mis à jour par Jung. Je veux indiquer par ceci que ce qui fait l'intérêt de la pensée jungienne, c'est qu'elle ne prétend pas inventer une nouvelle idéologie, une nouvelle explication du Monde et de l'humanité. Au contraire il s'est évertué pendant sa longue carrière à déterrer une sagesse immémoriale enfouie aussi bien dans la psyché de ses patients que dans les sous-bassements de notre civilisation. Il a fait un colossal travail d'archéologue, comme le préfigurait un de ses rêves de début de carrière, où il descendait des étages supérieurs de sa maison vers des caves de plus en plus profondes et de plus en plus anciennes, voire préhistoriques ${ }^{13}$.

Je peux résumer les éléments que j'ai cueillis dans cette jungle de l'œuvre jungienne en laissant à votre réflexion les propositions suivantes :

La psyché humaine est bisexuée.

Le jeu des oppositions entre le masculin et le féminin est également présent chez la femme et chez l'homme.

La différence tient à la prédominance des éléments qui sont actualisés dans la lumière de la conscience.

La féminité est principalement constituée par les traits suivants : familiarité avec les contenus de l'inconscient; tendance à conserver et protéger; énergie 
de croissance psychique; force d'intégration et de complétude de la personnalité.

En terminant je veux tout de même souligner que la femme moderne a développé une capacité admirable à utiliser ses ressources masculines. C'est probablement un des facteurs qui la place actuellement en situation de supériorité psychologique vis-à-vis de l'homme, avec aussi, sans doute, sa facilité à réagir aux tendances à l'unilatéralité : elle a un instinct de l'équilibre mental autrement plus vigoureux que celui de l'homme. Ce n'est pas étonnant que, dans les couples, ce soit toujours la femme qui sonne l'alarme quand la relation est en danger. Mais l'équilibre demeure toujours très difficile dans une société où la femme a dû se soumettre aux stéréotypes hypermasculins de la compétition, de l'agressivité, de la productivité et du froid calcul, pour arriver à se faire une place au soleil.

Quand les femmes auront gagné leur combat pour l'égalité, encore faudra$\mathrm{t}$-il que les valeurs féminines viennent peser de tout leur poids pour rétablir un équilibre gravement compromis par des millénaires de patriarcat. 